\title{
Characterization of multi-junction solar cells by mapping of the carrier transport efficiency using luminescence emission
}

\author{
Amaury Delamarre ${ }^{* 1,4}$, Jieyang Jia ${ }^{3}$, Paul Verdier ${ }^{1,4}$, Kentaroh Watanabe ${ }^{1,4}$, Masakazu Sugiyama ${ }^{2,4}$, \\ Yoshiaki Nakano ${ }^{2,4}$, Jean-François Guillemoles ${ }^{\dagger 1,4}$ \\ ${ }^{1}$ RCAST, The University of Tokyo, ${ }^{2}$ Department of Electrical Engineering and Information Systems, \\ The University of Tokyo, ${ }^{3}$ Stanford university, Department of electrical engineering, ${ }^{4} \mathrm{NextPV}$, LIA \\ CNRS-RCAST/U. Tokyo-U. Bordeaux
}

\begin{abstract}
Multijunction solar cells are currently the devices offering the largest conversion efficiencies of the solar radiation, which could be further increased by limiting their series resistances. A clear evaluation of the impact of those resistances is therefore required, and provided in this paper by introducing a mapping method of the current transport efficiency from luminescence images. This method brings finer information on the cell than electroluminescence methods, widely used so far for multi-junction cells, and offers much faster acquisition time than what could be obtained with a light beam induced current setup. While it has been theoretically and experimentally developed for single junction solar cells, its application to multijunction cells remains to be demonstrated. The purpose of this communication is to assess its validity and to explain some results that can be counterintuitive at a first sight. Two different triple-junction architectures are investigated and successfully compared with electrical measurements and calculations.
\end{abstract}

Keywords: transport efficiency, photovoltaic, multi-junctions, characterization, luminescence, reciprocity

\section{INTRODUCTION}

Multijunction solar cells (MJSC) are currently the devices offering the largest conversion efficiencies of the solar radiation (above $45 \%^{1,2}$ ). Among the different strategies to improve their characteristics, one would be to operate them at higher sunlight concentrations, current standards ranging from 500 to 1000 suns. Nevertheless, the maximum achievable concentration is limited by the various series resistances of the cells ${ }^{3}$ : window layer sheet resistance, metal / semiconductor resistance, tunnel junctions. Consequently, a careful design of the contact grid to reach the best tradeoff between series resistances and shadowing is necessary.

To assess the impact of the series resistances, measurement techniques evaluating the spatial transport efficiency are required. This can be done with a Light Beam Induced Current setup (LBIC) ${ }^{4}$, although this method has the drawback that it can be time consuming due to the necessity of scanning the surface. Another method by luminescence imaging ${ }^{5}$ is considered here. This method has been experimentally applied to a variety of single junctions (silicon ${ }^{6-8}, \mathrm{CIGS}^{9}, \mathrm{GaAs}^{10}$, perovskites ${ }^{11}$ ), but not to multijunction cells despite a real interest. The current communication aims at validating experimentally the method on such devices.

\section{THEORY}

The measurement of the transport efficiency by luminescence relies on the following reciprocity relation ${ }^{5}$ :

$$
f_{t}(x, y)=\left.\frac{\delta I_{T}}{\delta I(x, y)}\right|_{\delta V_{T}=0}=\left.\frac{\delta V(x, y)}{\delta V_{T}}\right|_{\delta I(x, y)=0}
$$

\footnotetext{
*delamarre@hotaka.t.u-tokyo.ac.jp

$\dagger$ jf.guillemoles@chimie-paristech.fr
}

Physics, Simulation, and Photonic Engineering of Photovoltaic Devices VI, edited by Alexandre Freundlich, Laurent Lombez, Masakazu Sugiyama, Proc. of SPIE Vol. 10099, 100990Z · C 2017 SPIE CCC code: $0277-786 X / 17 / \$ 18 \cdot$ doi: $10.1117 / 12.2250866$ 
$f_{t}(x, y)$ is the transport efficiency at a position $(x, y)$, defined as the ratio of the collected current $\delta I_{T}$ at the terminal to the local generation current $\delta I(x, y)$. This quantity is found to be equal to the ratio of the local diode voltage variation $\delta V(x, y)$ for a voltage variation at the terminal $\delta V_{T}$. An LBIC setup is able to measure directly the transport efficiency by measuring the middle element of equation (1) at any point on the surface of the cell. Alternatively, since the luminescence depends on the voltage applied to a solar cell, the third element of (1) can be determined by luminescence imaging. Assuming that the quasi-Fermi level splitting $\Delta \mu$ is constant in the depth of a cell, and therefore equal to its voltage $V$, the luminescence emission $\Phi(\mathrm{x}, \mathrm{y})$ can be described by the generalized Planck's law ${ }^{12}$ :

$$
\Phi(\mathrm{x}, \mathrm{y})=\int A(E) \frac{1}{4 \pi^{2} \hbar^{3} c^{2}}\left(\exp \left(\frac{E-q V(x, y)}{k T}\right)-1\right)^{-1} \cdot d E
$$

$A(E)$ is the absorption, $E$ the photon energy, T the temperature, while other constants have their usual signification. Since the term -1 in the brackets in the integral can be neglected, one can relate the luminescence image variation for a small variation of the terminal voltage $\delta V_{T}$ to the transport efficiency, thanks to the reciprocity relation (1):

$$
f_{t}(x, y)=\frac{\delta \ln (\Phi(x, y))}{q \delta V_{T} / k T}
$$

This has been theoretically proposed ${ }^{5}$, and used experimentally ${ }^{6-11}$. The validity of the deduced collection efficiency has been confirmed by comparing the collected current on single junctions $\left(\mathrm{GaAs}^{10}\right.$, silicon $\left.^{8}\right)$ to the spatially averaged value of the transport efficiency. Note that it has been observed, as expected, that the collection efficiency is reduced under increased illumination.

The reciprocity relation was demonstrated $i^{5}$ in two different manner: for a general arbitrary three dimensional geometry, then for a cell represented as an equivalent electrical network. This second demonstration is reused here in the case of the multijunctions. A multijunction cell can be represented by the equivalent circuit in Figure 1(a): lateral resistances represent the resistances of the top layer and any intermediate layers that can induce a lateral current, and the subcells are reprensented by diodes in parallel to current sources. Each diode can be in turn represented by two currents of ideality factors 1 and 2. Because we are considering small variation from a given working point (i.e. small variation of the generation $\delta I(x, y)$, or small variation of the applied voltage $\delta V_{T}$ ), each element can be represented by an equivalent differential resistance, as shown in Figure 1(b). Each resistance is equal to the inverse of the derivative of the I(V) characteristic of the element at the considered working point. The circuit can be further simplified by representing it as a two ports network, one for the terminal, one for the subcell $i$, at a position $(x, y)$, as in Figure 1(c). The reciprocity theorem in linear circuits ${ }^{13}$ allows us to write the reciprocity relation (1) for multijunction cells.

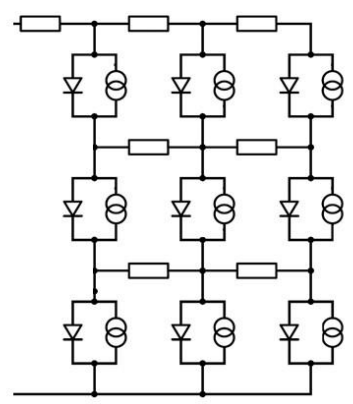

(a)

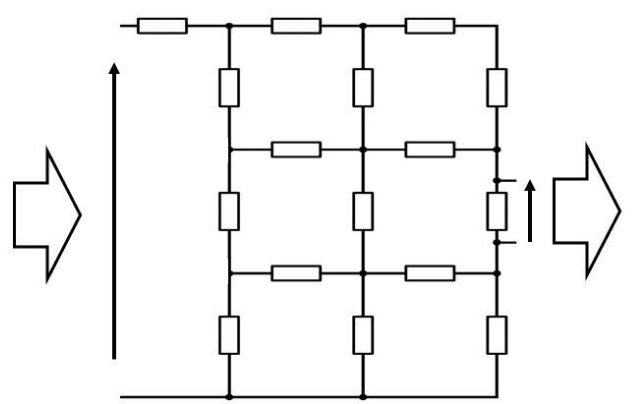

(b)

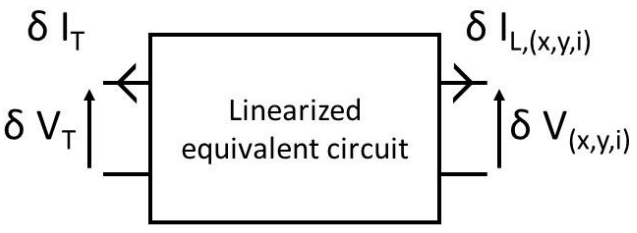

(c)

Figure 1 (a) Equivalent circuit of a multijunction solar cell. (b) Linearized equivalent circuit, in which each diode is replaced by a resistance representing its I(V) characteristic around a working point. (c) The complete linearized equivalent circuit can be considered as a quadrupole, the output representing the terminal, and a given position on the cell surface (x,y) for a given subcell (i). 


\section{EXPERIMENTAL SETUP}

Two different kinds of commercially available solar cells are considered here: lattice matched cells (InGaP / GaAs / Ge, samples A and B) and metamorphic cells (InGaP / InGaAs / Ge, sample C). Samples A and B are from the same supplier, but were differently processed and probably not from the same epitaxial growth. Three different light sources are used for each subcell: a $532 \mathrm{~nm}$ laser from Coherent (top cell), a $808 \mathrm{~nm}$ laser diode from Thorlabs (middle cell), and a xenon lamp from Asahi Spectra with a $990 \mathrm{~nm}$ long pass filter (bottom cell). The luminescence is collected in two different manner: either by a camera pco.1300 from pco, or with a Acton SP2300 spectrometer from Princeton instrument for spatially integrated signals. In any case, different sets of appropriate long pass and short pass filters are used in the detection, for cutting the excitation light source as well as selecting the emission of a single subcell. It is worth noting that only silicon detectors were used, so the luminescence signal of the bottom cell has not been observed. For electrical measurement of the collection efficiency, monochromatic illumination is obtained with a supercontinuum laser from fiannium and a lock-in SR510 from Stanford Research Systems. In any case, voltage is applied and current is measured with a 2602A Keithley source meter.

\section{RESULTS AND DISCUSSION}

The cells are firstly considered in the dark, i.e. the electroluminescence signal is recorded, for simplicity (4.1 and 4.2). Luminescence measurements under illumination bias are then considered (4.3).

\subsection{Electroluminescence measurement}

The electroluminescence signal of sample A has been measured, without spatial resolution. From this signal and applying the relation (3), a spatially averaged transport efficiency can be calculated, which is shown in Figure 2(a).

From a traditional approach, one would have expected the collection efficiency to be null in the dark. This is because even if one cell is illuminated, both other cells would remain unilluminated, and would therefore limit the collected current to zero. Nevertheless, we have to consider that only small departures from the working point are considered, which means that the additional illumination is negligible regarding the direct current in the device. In this condition, a non-zero collection efficiency is expected in the dark. This has been calculated in a one-dimensional equivalent circuit in Figure 2(b). The result of this calculation is shown in Figure 2(a) and reproduces the transport efficiency determined from luminescence.

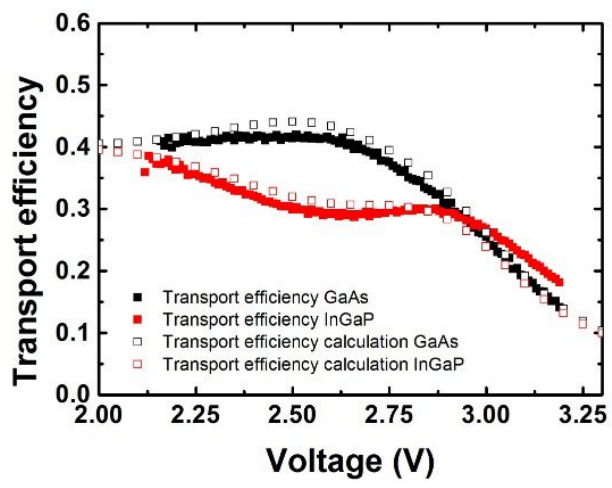

(b)

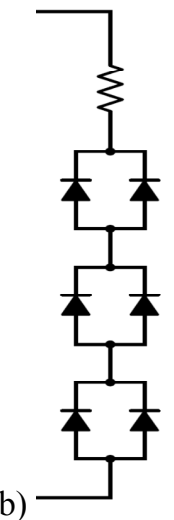

Figure 2 (a) Transport efficiency measured in electroluminescence as a function of the applied voltage. Experimental result and calculations are presented for both the top InGaP and the middle GaAs cell. The transport efficiency calculation is carried out considering the equivalent circuit of the cell presented in (b).

\subsection{Electroluminescence imaging}

The mapping capability of the method has been explored in the dark for the sample B. In Figure 3(a) is displayed an image of the cell surface, on which the contact grid can be observed. Along the axis perpendicular to those contacts, the averaged collection efficiency is extracted and plotted in Figure 3(b), for an applied voltage of $3.05 \mathrm{~V}$ (giving rise to a direct current equivalent to the short-circuit current that would be obtained under 144 suns). As in the case of the 
previous cell, we observe a collection efficiency different from zero. We also observe that the collection efficiency is not flat in the top cell, whereas it is independent on the position for the middle cell. We note that for moderate applied forward bias the collection efficiency is flat for both cells (not shown here).

The collection efficiency in the top cell is reduced in between two contacts. This might be understood qualitatively by the fact that the lateral current occurs in the emitter or the window layer of the top cell, which have a significant series resistance. On the opposite, large lateral currents can be sustained in between both top cells, where highly doped layers are inserted: the base of the top cell, the tunnel junction and the emitter of the middle cell. Such a behavior has been already observed in electroluminescence images of multijunction cells ${ }^{14,15}$. Nevertheless, the present measurement has the advantage that it gives directly its influence on the collection efficiency.

(a)

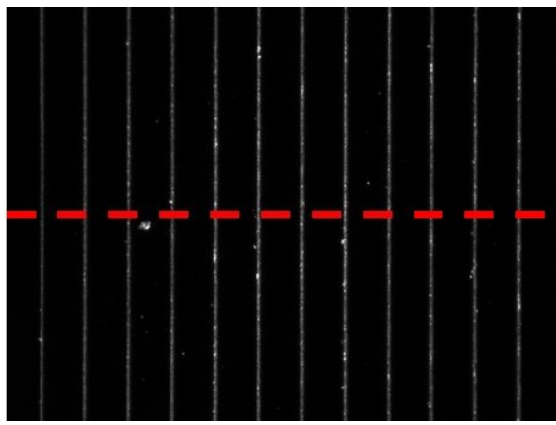

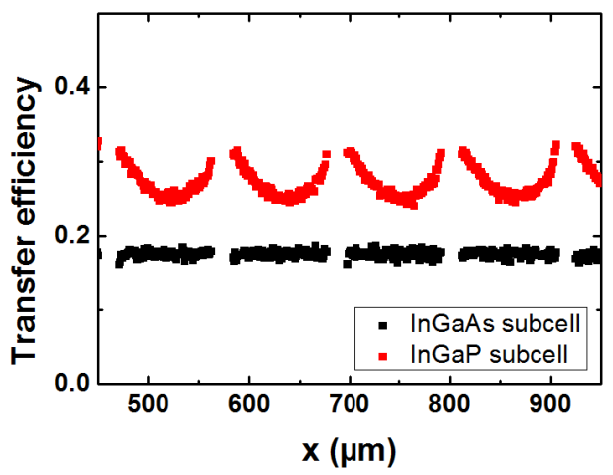

Figure 3 (b) Image of the cell surface, on which the contacts can be observed. Along the red dashed line are extracted the collection efficiency profiles plotted in (b).

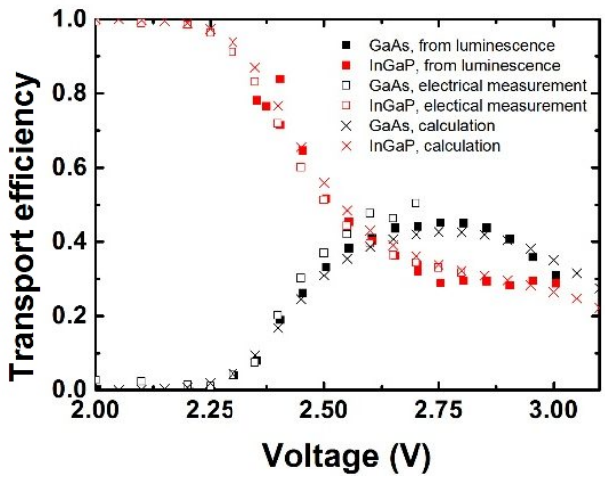

Figure 4 Collection efficiency as a function of voltage for a lattice matched triple junction, for illumination of the middle and bottom cell, while the top cell remains unilluminated. The values obtained using the luminescence method are compared to electrical measurements and simulation, using a similar equivalent circuit as the one presented in Figure 2 (a).

\subsection{Measurement under illumination}

Since the relevant collection efficiency should be measured under real operating condition, it is interesting to observe how it behaves under illumination. Sample $\mathrm{C}$ has been investigated with illumination of the middle and the bottom cell, while the top cell remained in the dark. After luminescence measurement, the surface average of the collection efficiency is plotted in Figure 4 as a function of applied voltage. Under the same condition, the electrical collection efficiency measured with a lock-in is plotted, as well as the result of calculation in an equivalent circuit (Figure 2(b)).

We observe that both measurements show the same voltage variation, which is reproduced with calculation. At low biases, the collection efficiency of the limiting cell is close to one, whereas the collection efficiency of the middle cell 
remains at zero. This situation changes at large applied voltage, where the collection of both cells takes positive values ${ }^{16}$. For even larger voltages, the collection efficiency is reduced due to series resistances.

Although both measurements agree, it is interesting to note that the voltage range in which they can be measured is different. On the one hand, the luminescence signal depends exponentially on the quasi-Fermi level splitting. This means that a minimum voltage is required for a reasonable signal to noise ratio. In our case, we could not measure the luminescence of the top cell below $2.35 \mathrm{~V}$. On the other hand, the electrical measurement needs to probe a current variation within a background signal that increases exponentially with the voltage. In our case, it became difficult to measure values above $2.7 \mathrm{~V}$.

\section{CONCLUSION}

This communication aims at measuring the current collection efficiency in multijunction cells. This is a relevant quantity since the maximum concentration under which they can be operated is limited by series resistances. For a better evaluation of this mechanism, a recently developed method for mapping the collection efficiency is explored. Two different device architectures were investigated in three configurations. Firstly, the surface averaged collection efficiency has been measured in the dark, which could be reproduced by calculation in an equivalent circuit. Secondly, mapping of the collection has been performed in the dark. We could evidence a different behavior of the top and the middle cell, which can be attributed to conductive layers inserted between the top and the middle cell. Finally, the spatially averaged collection efficiency has been measured under selective illumination of the middle and the bottom cell. Those values agree well with electrical measurement of the current under the same condition and calculations, for different applied voltages. Those three experiments validate the method, while showing its relevance for multijunction cell characterization.

\section{ACKNOWLEDGEMENTS}

A part of this study was supported by NEDO project: the research and development of ultra-high efficiency and low-cost III-V compound semiconductor solar cell modules. The authors are grateful to Solar Junction Corporation for sharing samples.

\section{REFERENCES}

[1] Green, M. A., Emery, K., Hishikawa, Y., Warta, W., Dunlop, E. D., Levi, D. H.., Ho-Baillie, A. W. Y., "Solar cell efficiency tables (version 49)," Prog. Photovolt. Res. Appl. 25(1), 3-13 (2017).

[2] "New world record for solar cell efficiency at 46\% - Fraunhofer ISE.", Fraunhofer Inst. Sol. Energy Syst. ISE, $<$ http://www.ise.fraunhofer.de/en/press-media/press-releases/2014/new-world-record-for-solar-cell-efficiency-at46-percent.html> (7 February 2017 ).

[3] Ochoa, M., Barrigón, E., Barrutia, L., García, I., Rey-Stolle, I.., Algora, C., "Limiting factors on the semiconductor structure of III-V multijunction solar cells for ultra-high concentration (1000-5000 suns)," Prog. Photovolt. Res. Appl. 24(10), 1332-1345 (2016).

[4] Carstensen, J., Popkirov, G., Bahr, J.., Föll, H., "CELLO: an advanced LBIC measurement technique for solar cell local characterization,” Sol. Energy Mater. Sol. Cells 76(4), 599-611 (2003).

[5] Wong, J.., Green, M., "From junction to terminal: Extended reciprocity relations in solar cell operation," Phys. Rev. B 85(23), 235205 (2012).

[6] Wong, J., Sridharan, R., Wang, Y. C.., Mueller, T., "Differential electroluminescence imaging and the current transport efficiency of silicon wafer solar cells," Photovolt. Spec. Conf. PVSC 2014 IEEE 40th, 0975-0979, IEEE (2014).

[7] Rau, U., Huhn, V., Stoicescu, L., Schneemann, M., Augarten, Y., Gerber, A.., Pieters, B. E., "Photocurrent collection efficiency mapping of a silicon solar cell by a differential luminescence imaging technique," Appl. Phys. Lett. 105(16), 163507 (2014).

[8] Huhn, V., Pieters, B. E., Augarten, Y., Gerber, A., Hinken, D.., Rau, U., "Imaging photocurrent collection losses in solar cells," Appl. Phys. Lett. 109(22), 223502 (2016). 
[9] Gerber, A., Huhn, V., Tran, T. M. H., Siegloch, M., Augarten, Y., Pieters, B. E.., Rau, U., “Advanced large area characterization of thin-film solar modules by electroluminescence and thermography imaging techniques," Sol. Energy Mater. Sol. Cells 135, 35-42 (2015).

[10] Delamarre, A., Lombez, L., Watanabe, K., Sugiyama, M., Nakano, Y.., Guillemoles, J.-F., "Experimental Demonstration of Optically Determined Solar Cell Current Transport Efficiency Map,” IEEE J. Photovolt. 6(2), 528-531 (2016).

[11] El-Hajje, G., Momblona, C., Gil-Escrig, L., Ávila, J., Guillemot, T., Guillemoles, J. F., Sessolo, M., Bolink, H. J.., Lombez, L., "Quantification of spatial inhomogeneity in perovskite solar cells by hyperspectral luminescence imaging," Energy Env. Sci (2016).

[12] Würfel, P., "The chemical potential of radiation,” J. Phys. C Solid State Phys. 15, 3967-3985 (1982).

[13] Tellegen., "A general network theorem, with applications," Phillips Res Rep 7, 259 (1952).

[14] Nesswetter, H., Lugli, P., Bett, A. W.., Zimmermann, C. G., "Electroluminescence and Photoluminescence Characterization of Multijunction Solar Cells," IEEE J. Photovolt. 3(1), 353-358 (2013).

[15] Nesswetter, H., Dyck, W., Lugli, P., Bett, A. W.., Zimmermann, C. G., "Luminescence based series resistance mapping of III-V multijunction solar cells," J. Appl. Phys. 114(19), 194510 (2013).

[16] Meusel, M., Baur, C., Létay, G., Bett, A. W., Warta, W.., Fernandez, E., "Spectral response measurements of monolithic GaInP/Ga (In) As/Ge triple-junction solar cells: Measurement artifacts and their explanation,” Prog. Photovolt. Res. Appl. 11(8), 499-514 (2003). 